\title{
Focus on Mobile Phone on Human Movement in Public Space
}

\author{
Yuki Ogimoto and Kazunari Tanaka
}

\begin{abstract}
The purpose of this study is to find the relationship between the characteristics of space and pedestrian behaviors, to grasp the relationship between the place of daily, everyday actions, including mobile phone usage actions and the image of the surrounding space. This would ultimately lead to designing by taking human behavior into consideration. The authors start by extracting external factors that appear to be affecting people. Furthermore, the authors will calculate specific figures and analyze them. Analysis is based on the relationship between the elements of the spatial shape extracted by physical surveying, image analysis, as well as human attributes. The survey was conducted at an open space around Osaka station. The authors found that the positions of mobile phone operation varies depending on the time of usage. For this study, we chose a place where smartphones are easily and commonly used.
\end{abstract}

Keywords-Mobile phone, Pavement, Open space, Public space

\section{INTRODUCTION}

The purpose of this research is to design pavement taking pedestrians into consideration, especially the elderly, which is the fastest growing age group. In recent years, it is considered that the walking paths of people are changing due to the design of sidewalks and the walking environment. By analyzing the elements of the spatial shape, the authors believe that they can utilize this for urban planning, by clarifying the route according to an individual's age. In previous studies, walkability tests and analysis were performed for each type of pavement. There are few studies on public spaces. If it is possible to grasp the respective walking route by attributes such as age, then this can lead to improved functional design universally. Designers are trying to create spaces that various people can use easily, however, there are actually various aspects to consider for design.

\section{PURPOSE AND METHOD OF RESEARCH}

The authors identify the relationship between the characteristics of space shape and pedestrian features, and grasp the relationship pertaining to the place of each action and the shape of the surrounding space. It is ultimately best to propose design taking into consideration human behavior.

In this research, the authors will quantitatively find the influence of invisible design, utilizing physical and pedestrian behavior data obtained from a field survey.

Ultimately, the authors will refine it to detailed analysis that

Yuki Ogimoto, Osaka Institute of Technology, Japan

Kazunari Tanaka, Osaka Institute of Technology, Japan connects how space design affects pedestrian influence, while acquiring a large amount of pedestrian behavior data. In order to grasp and express the influence of the spatial shape and the influence range, the authors extract external factors that appear to be affecting people. Furthermore, the authors will produce specific figures and analyze them, analysis is made on the relationship between the elements of the spatial shape extracted by the survey and human attributes.

\section{POINT OF INTEREST}

In conducting this research, the authors focus on mobile phones and public spaces. Currently, mobile phones are spreading globally, with the advent of smartphones from 2010, it has become an indispensable tool to our daily lives. However, mobile phone related accidents are also occurring.

The graph below shows the penetration rate of communication terminals in Japan (Figure 1).

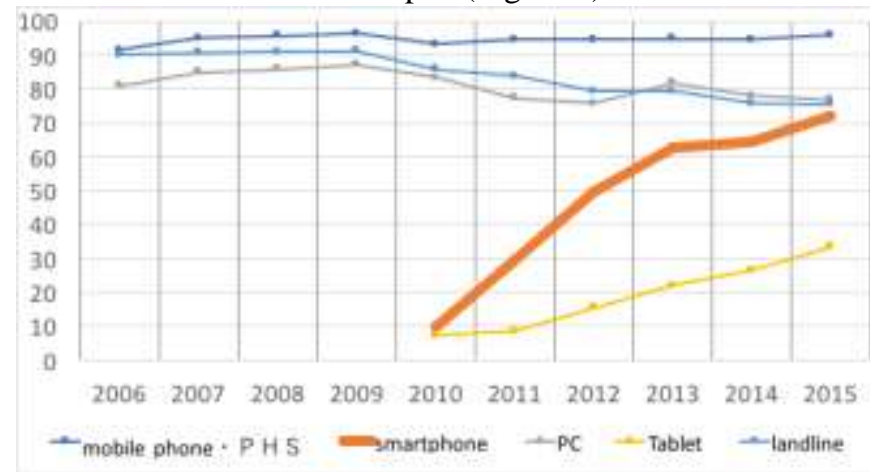

Fig. 1 Japanese communication terminal of Penetration

\section{TARGET AREA}

For the target area, the authors wanted to use a space that had various features common in public places such as; pillars, walls, stairs, monuments etc.

Osaka is one of the most dense urban areas in Japan. The number of tourists is the highest every year, centered in Osaka, many tourist attractions are actively using the public space. Therefore, the target was the Umeda area, which is considered to be one of the central areas in Osaka (Figure 2, 3).

In this research, the authors covered a space used mainly by pedestrians. A preliminary survey was conducted in the open space around Osaka station, and a place considered to be similar was set as the target site. In addition, this survey site is a place with many railway stations. 


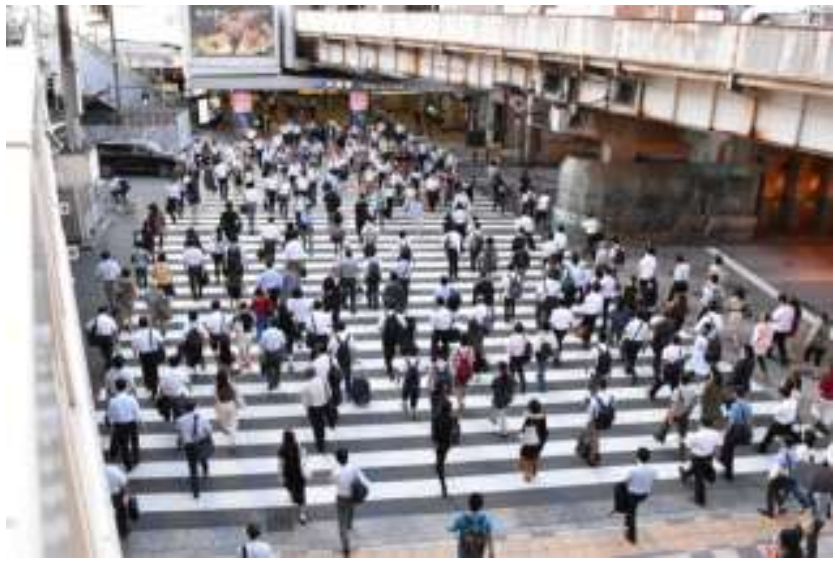

Fig. 2 Pedestrian traffic at Osaka Station

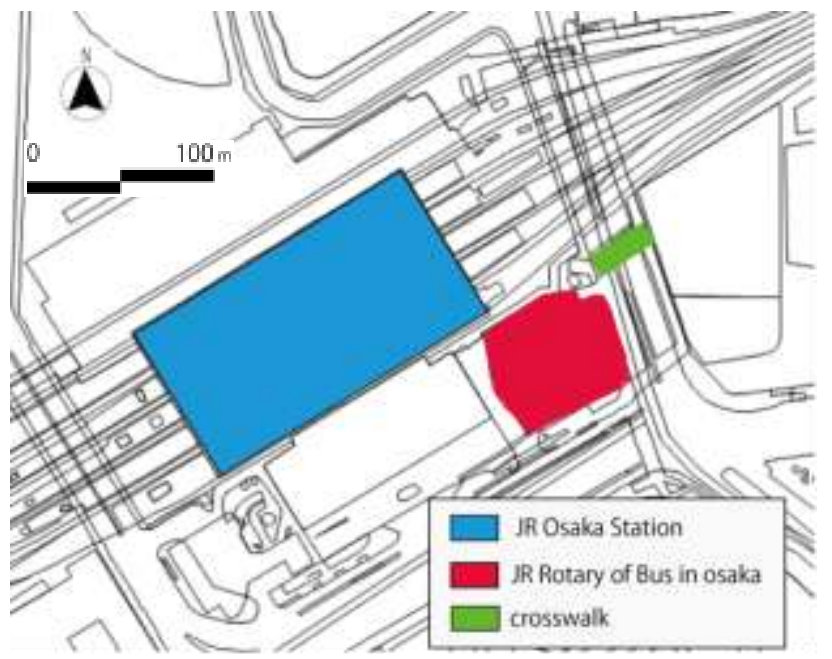

Fig. 3 Target Area

\section{INVESTIGATION AND ANALYSIS}

In this research, we can understand the usage situation by finding places where mobile phones are used. For that purpose, we photographed the target location.

\section{A. Investigation}

First, the authors took the photos to capture the location and constituent elements of the people, then plotted this on GIS (Figure 4,5).

The figure below shows a smartphone operator and non-operator. The shooting point is indicated by a 60 -degree cone. The survey summary is shown in Table 1.

TABLE I

SURVEY SUMMARY

\begin{tabular}{|l|l|}
\hline Schedule & $\begin{array}{l}\text { 2017 August 30 (Wednesday), } \\
\text { September 1 (Friday) }\end{array}$ \\
\hline Time frame & $8: 20-9: 00$ \\
\hline weather & Cloudy weather \\
\hline Target area & osaka station \\
\hline Method & Photo shooting \\
\hline
\end{tabular}

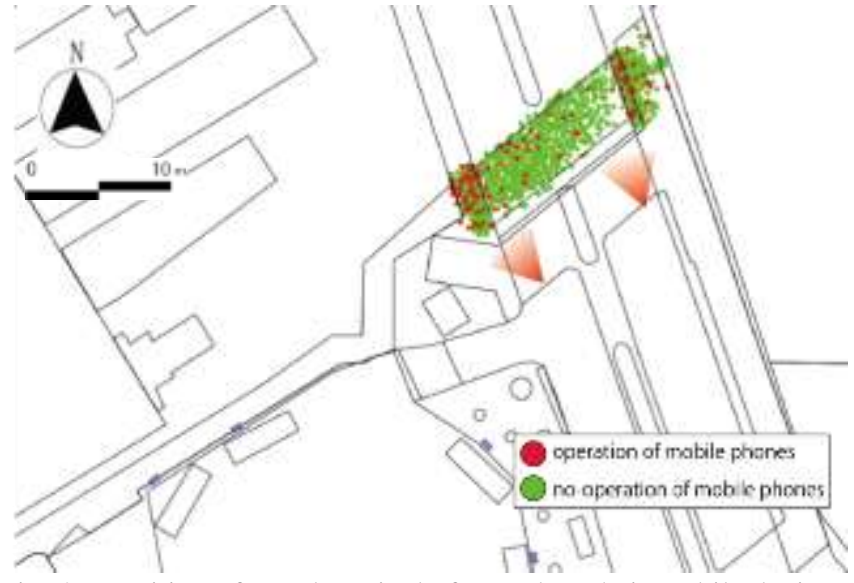

Fig. 4 Position of people actively focused on their mobile devices (crosswalk)

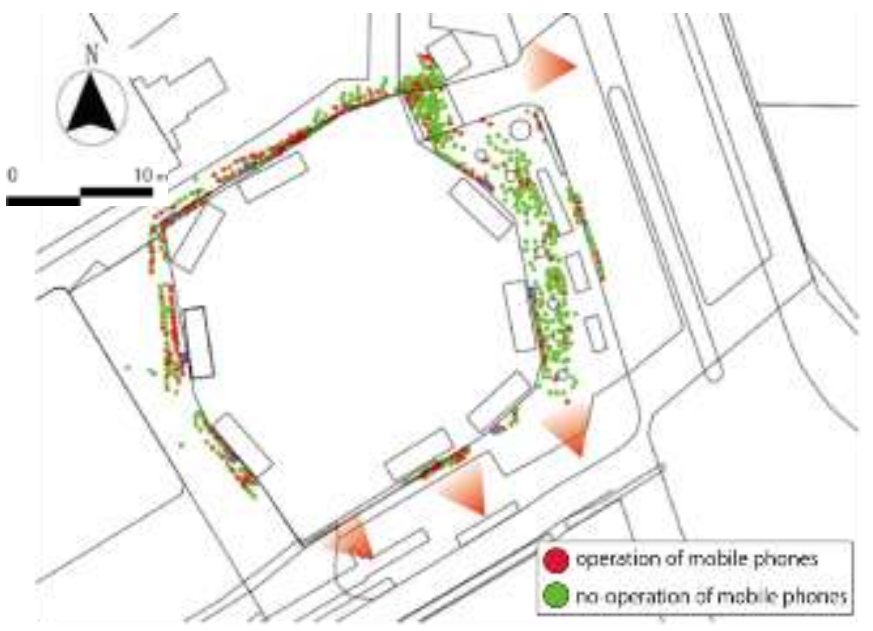

Fig. 5 Position of people actively focused on their mobile devices (Bus Rotary)

\section{Result (1)}

Next, from the obtained results, we calculated where people concentrate (Figure 6). The method of analysis was done by density estimation. The red area shows a high numerical value. This method can reduce the error in the case of representing the concentrated area of people. This has been proved by a previous study (References 3).

In addition, there were two types of behavior for people who operated mobile phones; 1) operates while not walking 2) operates while walking. 


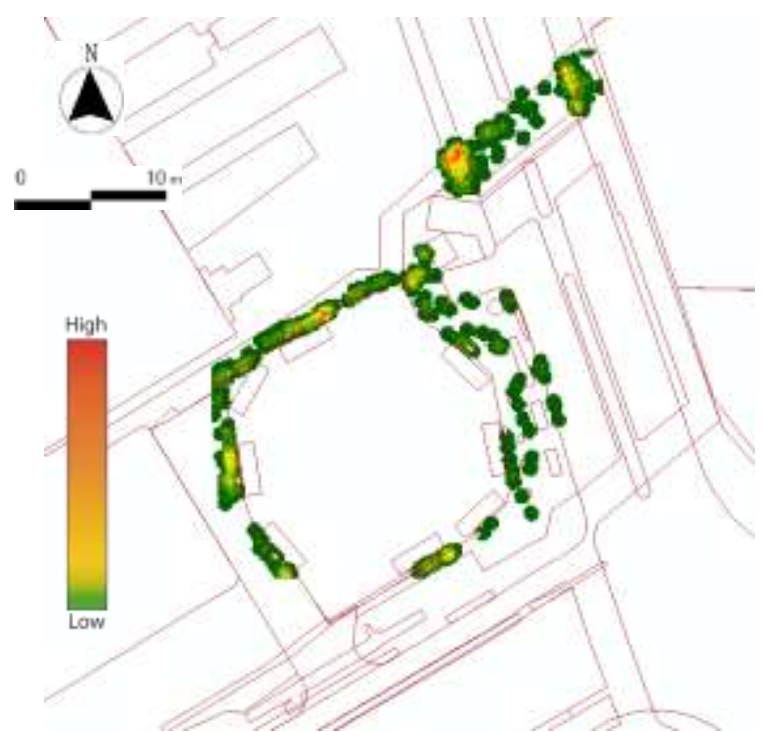

Fig. 6 This is the example for figure formatting

\section{Analysis (1)}

Based on the above results, we analyzed the two kinds of behavior separately. This was carried out at the same density point as before.

The place which shows the highest numerical value from the first graph is in front of the pedestrian crossing (Figure 7), and the place with the highest numerical value of the second graph is also the pedestrian crossing (Figure 8).

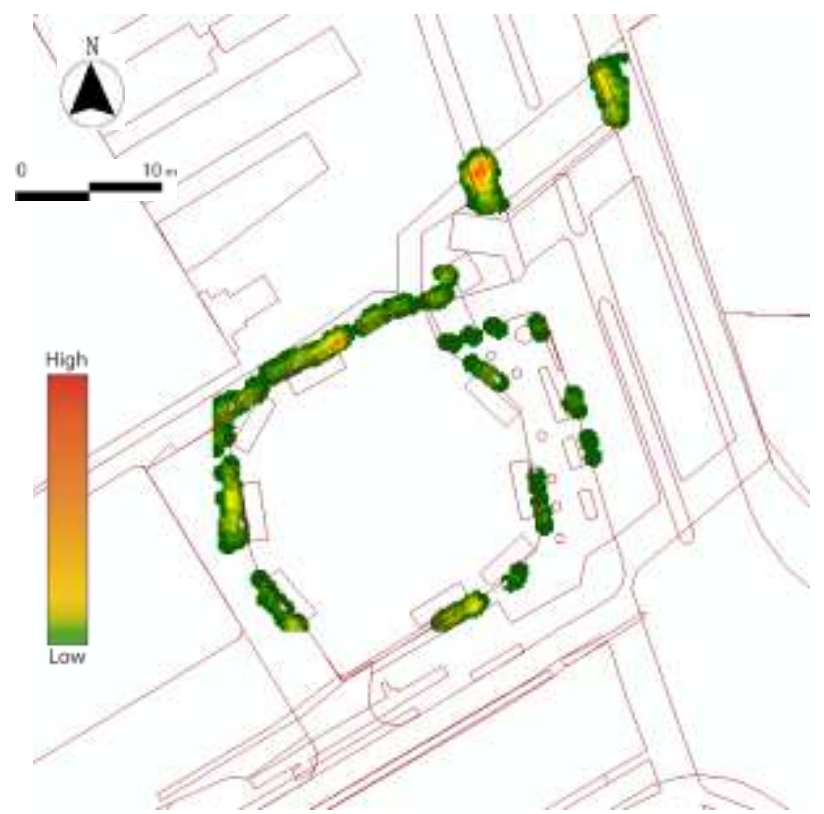

Fig. 7 Operates mobile phone without walking

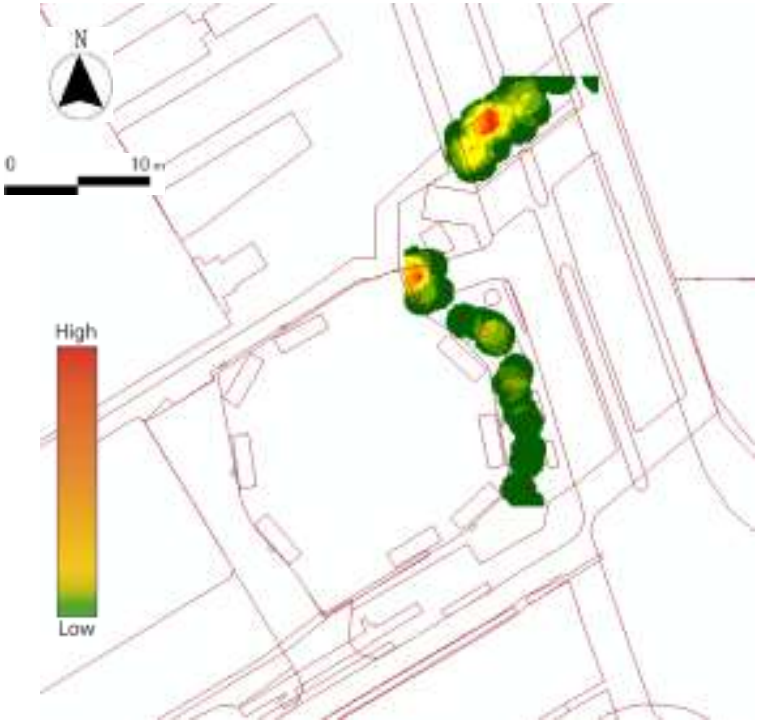

Fig. 8 Operates mobile phone while walking

\section{Consideration}

The results commonly showed that usage at the pedestrian crossing was high. Therefore, the authors considered it necessary to investigate the location of the pedestrian crossing in further detail.

\section{Investigation (2)}

The purpose of this survey is for detailed analysis of pedestrian crossings.

The outline of the survey is shown in Table 2. The survey method was filming the pedestrian flow. The shooting time was 1 minute and 40 seconds. It is 1 minute 30 seconds between the red signal and 10 seconds for the blue signal. Also, the author conducted this survey three times.

TABLE II

SURVEY SUMMARY

\begin{tabular}{|l|l|}
\hline Schedule & 2017 August 23 (Wednesday) \\
\hline Time frame & $8: 30-9: 00$ \\
\hline weather & Cloudy weather \\
\hline Target area & crosswalk \\
\hline Method & Movie shooting \\
\hline
\end{tabular}

\section{Result (2)}

The results are shown in the below figure. In this figure, the authors marked persons who operate a mobile phone and those who don't (Figure 9).

The author will compile these results. 


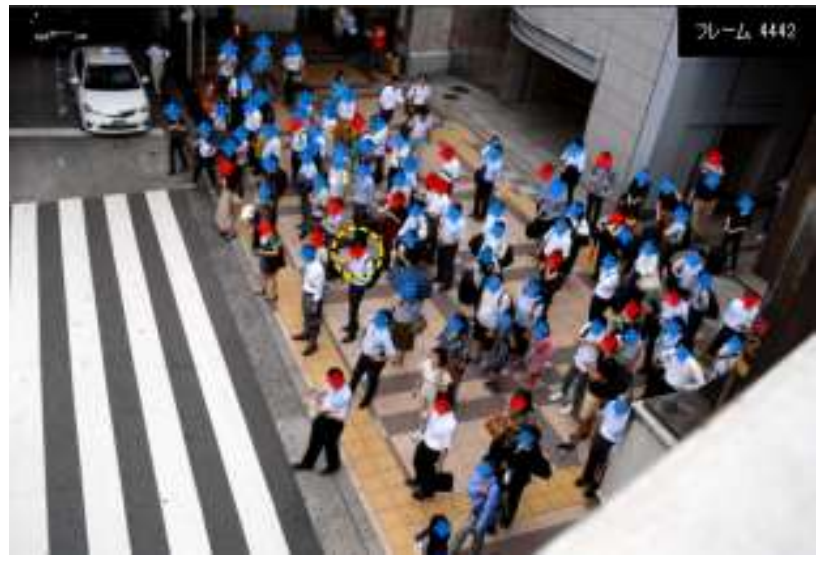

Fig. 9 Pedestrian flow at Osaka station

TABLE III

OPERATORS OF MOBILE PHONES AT CROSSWALKS

\begin{tabular}{|l|c|}
\hline Contents & Number of people \\
\hline Operator of mobile phone & 145 \\
\hline Non-operator of mobile phone & 370 \\
\hline Combined & 515 \\
\hline
\end{tabular}

\section{Analysis (2)}

From the calculated results, the proportion of people who operate mobile phones while walking was calculated (Table 4). All proportions were calculated based on the number of pedestrians caught in the vicinity of the pedestrian crossings. The purpose of this analysis is to clarify, as rising situation of pedestrians walking while using their mobile phones is becoming a social problem.

TABLE IV

PERCENTAGE OF OPERATORS OF MOBILE PHONES AT PEDESTRIAN CROSSINGS

\begin{tabular}{|l|c|}
\hline Contents & Proportion $(\%)$ \\
\hline Operator of mobile phone (515) & 28.2 \\
\hline $\begin{array}{l}\text { People who start to operate mobile } \\
\text { phones while walking(515) }\end{array}$ & 13.0 \\
\hline $\begin{array}{l}\text { Percentage of mobile phone operators } \\
\text { walking smashing (145) }\end{array}$ & 46.2 \\
\hline
\end{tabular}

\section{Consideration}

The place to operate the smartphone is a place to forcibly stop walking.

Therefore, it is considered to be a place where pedestrians make time to operate mobile phones.

\section{CONCLUSION}

In this research, the authors focused attention on pedestrians in public spaces in the city and observed and analyzed them. From the survey, we were able to grasp those who used cell phones in public spaces.

In addition, analysis clarified where it concentrates. When touching a mobile phone at a pedestrian crossing, $46.2 \%$ of the people found that they used the mobile phone while walking, The above results were discussed.

\section{REFERENCES}

[1] M. Kousuke, H. Katuyuki, T.Kazunari, Y.Shin , Civil Engineering Association Kansai Branch Annual Scientific Lecture Summary IV-19 2015

[2] T.Yanobe, T.Kazunari, Y.Shin, STUDY DOWNTOWN STVANGER TO THE PEDESTRIAN SPACE 25.A-3-3(CD)

[3] S.Takahiro, M.Takuya Analyzing Visitors' Activity Area in Downtown Kumamoto using Smartphone-based Travel-Survey Date Journal of the City Planning Institute of Japan, Vol.50 No.3, October,2015.

[4] Y.Yurika, H.Kazuto, W.Hitoshi, Spatial usage behavior by age in station concourse pp651 pp652 2012 